ARTICLE

Received 6 Sep 2014 | Accepted 16 Jan 2015 | Published 20 Feb 2015

DOI: $10.1038 /$ ncomms 7310

OPEN

\title{
An octave-spanning mid-infrared frequency comb generated in a silicon nanophotonic wire waveguide
}

\author{
Bart Kuyken ${ }^{1,2}$, Takuro Ideguchi ${ }^{3}$, Simon Holzner ${ }^{3,4}$, Ming Yan ${ }^{3,4}$, Theodor W. Hänsch ${ }^{3,4}$, Joris Van Campenhout ${ }^{5}$, \\ Peter Verheyen ${ }^{5}$, Stéphane Coen ${ }^{6}$, Francois Leo ${ }^{1,2}$, Roel Baets ${ }^{1,2}$, Gunther Roelkens ${ }^{1,2}$ \& Nathalie Picqué ${ }^{3,4,7}$
}

Laser frequency combs, sources with a spectrum consisting of hundred thousands evenly spaced narrow lines, have an exhilarating potential for new approaches to molecular spectroscopy and sensing in the mid-infrared region. The generation of such broadband coherent sources is presently under active exploration. Technical challenges have slowed down such developments. Identifying a versatile highly nonlinear medium for significantly broadening a mid-infrared comb spectrum remains challenging. Here we take a different approach to spectral broadening of mid-infrared frequency combs and investigate CMOScompatible highly nonlinear dispersion-engineered silicon nanophotonic waveguides on a silicon-on-insulator chip. We record octave-spanning (1,500-3,300 nm) spectra with a coupled input pulse energy as low as $16 \mathrm{pJ}$. We demonstrate phase-coherent comb spectra broadened on a room-temperature-operating CMOS-compatible chip.

\footnotetext{
${ }^{1}$ Photonics Research Group, Department of Information Technology, Ghent University-imec, Sint-Pietersnieuwstraat 41, 9000 Ghent, Belgium. ${ }^{2}$ Center for Nano- and Biophotonics (NB-Photonics), Ghent University, 9000 Ghent, Belgium. ${ }^{3}$ Max Planck Institut für Quantenoptik, Hans-Kopfermannstrasse 1, 85748 Garching, Germany. ${ }^{4}$ Ludwig-Maximilians-Universität München, Fakultät für Physik, Schellingstrasse 4/III, 80799 Munich, Germany. ${ }^{5}$ imec, Kapeldreef 75, 3001 Leuven, Belgium. ${ }^{6}$ Department of Physics, The University of Auckland, Private Bag 92019 Auckland, New Zealand. ${ }^{7}$ Institut des Sciences Moléculaires d'Orsay, CNRS, Bâtiment 350, 91405 Orsay, France. Correspondence and requests for materials should be addressed to N.P. (email: nathalie.picque@mpq.mpg.de) or to B.K. (email: Bart.Kuyken@intec.ugent.be).
} 
F requency combs in the mid-infrared region ${ }^{1}$ have been mostly generated by nonlinear frequency conversion of near-infrared frequency combs. Although the field is currently very active with the exploration of many different and promising approaches ${ }^{2-4}$, producing a very broad spectrum with a slowly varying envelope remains challenging. Supercontinuum generation in a highly nonlinear fibre is known, under certain circumstances $^{5}$, to be a powerful way to generate an octavespanning frequency comb.

However, in the mid-infrared spectral region, suitable materials have remained scarse and difficult to engineer. Phase-coherent octave-spanning frequency comb generation has been achieved by spectral broadening of optical parametric oscillators (OPOs) ${ }^{6}$ and thulium-doped fibre laser ${ }^{7-9}$ frequency combs in nonlinear chalcogenide-tapered fibres. Taper lifetimes have recently been improved to several days with hybrid silica-chalcogenide structures, in which octave-spanning frequency comb generation has been reported ${ }^{8,9}$ using 65 -fs pulses of only 18 pJ. Promising solutions for enhanced stability are presently under investigation with multimaterial chalcogenide nanotapers ${ }^{10}$. Another approach is the use of quasi-phase-matched periodically poled lithium niobate (PPLN) waveguides. Impressive results have been obtained and an octave-spanning phase-coherent supercontinuum has been generated ${ }^{11}$. However, absorption between 2,500 and 2,800 $\mathrm{nm}$, and more importantly the limited transparency of lithium niobate beyond $4,500 \mathrm{~nm}$, inhibits the scaling of the technology to longer wavelengths. Furthermore, high-energy pulses $(7 \mathrm{~nJ})$ are needed because of the moderate nonlinearity of the waveguide. In addition, during the poling of the crystal small random variations on the location of the walls of the poled domains are introduced. This aberration increases the conversion of parasitic processes significantly ${ }^{11,12}$ and makes modelling difficult.

Silicon-based waveguides have been originally conceived for the telecommunication region. In this region, octave-spanning supercontinuum generation has been demonstrated by pumping silicon nitride waveguides with $150-\mathrm{pJ}$ pulses centred at $1.3 \mu \mathrm{m}$ (ref. 13), but the coherence conservation in the supercontinuum generation process has not been investigated. Recently, the application of silicon technology to the mid-infrared spectral region has attracted significant interest ${ }^{14-16}$. Silicon nanophotonic wire waveguides can be engineered ${ }^{17}$ within a nanometre precision in a standard CMOS facility. Such waveguides offer many advantages for mid-infrared nonlinear optics, mostly related to the wide transparency range of silicon $(1.1-8 \mu \mathrm{m})$, its high nonlinear refractive index, the possibility of precise dispersion engineering of the waveguide platforms and the high refractive index contrast between the silicon waveguide core and the cladding material (typically, $\mathrm{SiO}_{2}$ or air), which allows for densely integrated waveguide systems with a nonlinear parameter one to two orders of magnitude higher than that possible in the chalcogenide or silicon nitride systems.

In this article, we report on the design of strongly nonlinear, dispersion-controlled silicon photonic wire waveguides. We harness such chemically stable waveguides for mid-infrared supercontinuum generation, and we demonstrate a phasecoherent frequency comb generator with a $30-\mathrm{dB}$ bandwidth spanning from 1,540 up to $3,200 \mathrm{~nm}$, with coupled input pulse energies as low as $16 \mathrm{pJ}$.

\section{Results}

A highly nonlinear dispersion-engineered silicon waveguide. The photonic wire is fabricated in a CMOS pilot line ${ }^{17}$ on a $200-\mathrm{mm}$ silicon-on-insulator (SOI) wafer and consisting of a $390-\mathrm{nm}$-thick silicon device layer on top of a $2-\mu \mathrm{m}$ buried oxide layer. The inset in Fig. 1a) shows a schematic cross-section of the silicon photonic wire. The 1-cm-long air-clad photonic wire has a rectangular cross-section of $1,600 \mathrm{~nm} \times 390 \mathrm{~nm}$. The waveguide is slightly over etched by $10 \mathrm{~nm}$ into the buried oxide. The photonic wire widens near the cleaved facets to a $3-\mu \mathrm{m}$ wide waveguide section for improved coupling efficiency. As a result of the high nonlinear index of silicon ${ }^{18}$ and the strong optical confinement obtained by the high linear refractive index of silicon, the nonlinear parameter in the silicon wire is $38(\mathrm{Wm})^{-1}$ at $2,300 \mathrm{~nm}$ for the highly confined quasi-TE mode. Such a high nonlinear parameter in silicon waveguides shows the advantage of using silicon over chalcogenide tapers $\left(\gamma=4.5(\mathrm{Wm})^{-1}\right.$ (ref. 6)) and silicon nitride waveguides $\left(\gamma=1.2(\mathrm{Wm})^{-1}\right.$ (ref. 13)) where the nonlinear parameter is much lower. As a result of the high confinement, the waveguide dispersion of the silicon photonic wire contributes strongly to the overall dispersion of the optical waveguide, such that group velocity dispersion can be engineered by optimizing the waveguide dimensions. The group velocity dispersion of the quasi-TE mode of the dispersionengineered photonic wire waveguide as a function of wavelength is shown in Fig. 1a. The group velocity dispersion is simulated with the help of a finite element mode solver (Fimmwave). The zero-dispersion wavelength is at $2,180 \mathrm{~nm}$ and the dispersion becomes positive (normal) at shorter wavelengths, while the dispersion remains low over a wide spectral band. By using a cut-back technique the propagation loss for the quasi-TE mode is determined to be $<0.2 \mathrm{~dB} \mathrm{~cm}^{-1}$ in the wavelength range of 2,200-2,400 nm.
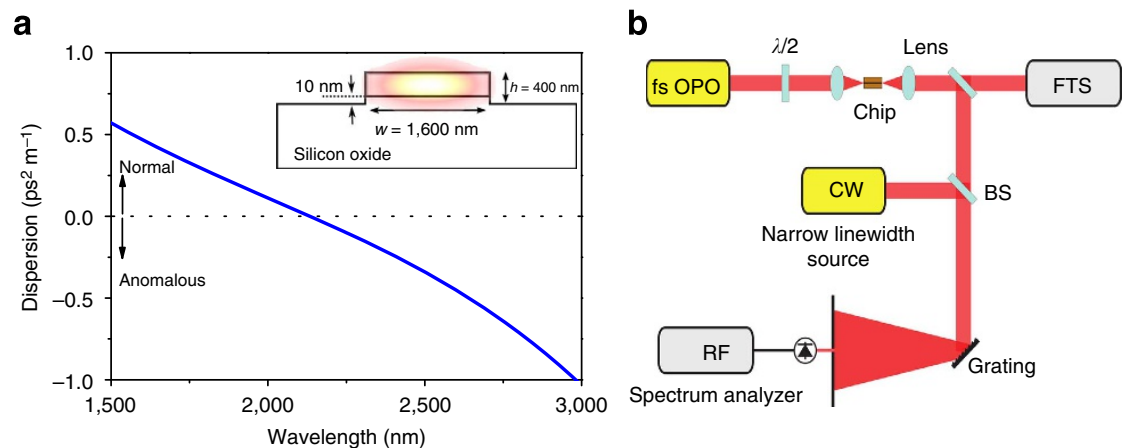

Figure 1 | The simulated dispersion of the photonic wire waveguide and the experimental set-up. (a) The zero-dispersion wavelength of the quasi-TE mode is at 2,180 nm, while the dispersion is normal at shorter wavelengths and anomalous at longer wavelengths. The waveguide cross-section is shown in the inset. (b) Experimental set-up: the OPO pumped by a Ti-Sapphire mode-locked laser is coupled to the silicon chip with a lens. The output of the chip can be sent to a photodetector or a spectrometer. 
The experimental set-up for supercontinuum generation. The set-up is shown in Fig. 1b. The frequency comb seed source consists of a homemade mid-infrared singly resonant $\mathrm{OPO}^{19}$ at a repetition frequency of $100 \mathrm{MHz}$, synchronously pumped by a femtosecond mode-locked Ti-Sapphire laser. The OPO is tuned to a centre wavelength of $2,290 \mathrm{~nm}$, close to the zero-dispersion wavelength of $2,180 \mathrm{~nm}$ of the silicon waveguide. Pumping a waveguide close to the zero-dispersion wavelength in the anomalous region allows for broadband supercontinuum generation ${ }^{5}$. The OPO has a pulse duration of $70 \mathrm{fs}$ (see Supplementary Fig. 1), while its average power is $35 \mathrm{~mW}$. The ultrashort mid-infrared fs pulses coming from the OPO are coupled to the quasi-TE mode of the silicon photonic wire using a high (Numerical Aperture) NA $(\mathrm{NA}=0.85)$ chalcogenide lens with a focal length of $1.87 \mathrm{~mm}$ (see Supplementary Methods for details). The output of the chip is coupled, using another chalcogenide lens, to a Fourier transform spectrometer to quantify the spectrum of the output pulses. The coupling loss at the input waveguide facet is estimated to be $12 \mathrm{~dB}$, leading to an on-chip peak power of $225 \mathrm{~W}$ or pulse energy of $16 \mathrm{pJ}$. The high coupling loss at the waveguide facet stems from the bad overlap of the quasi-TE mode of the waveguide and the mode profile at the focus plane of the lens. However, spot size converters ${ }^{20}$ could be used to significantly improve the coupling efficiency. We note that the coupled pulse energy and pulse duration that we use are similar to that used in ref. 8 for phase-coherent supercontinuum generation in a chalcogenide-silica hybrid waveguide.

Spectral broadening in a silicon photonic nanowire waveguide. The spectra at the input and output of the waveguide are shown in Fig. 2) for a pulse energy of $16 \mathrm{pJ}$. Spectra at lower pulse energies can be found in Supplementary Fig. 2. The spectrum of the pulses is significantly broadened in the silicon photonic wire waveguide and spans more than an octave: the $30-\mathrm{dB}$ bandwidth spans from 1,540 up to $3,200 \mathrm{~nm}$ at the output. The peak at $1,600 \mathrm{~nm}$ is located in the normal dispersion regime of the waveguide and is generated through dispersive wave generation, a method used to spectrally extend a supercontinuum ${ }^{16}$. In the course of the several weeks of experimental investigations, we did not observe any modification of the characteristics of the supercontinuum at the output of the silicon waveguide. Consistently, SOI platforms are used in electronics ${ }^{21}$ and optics $^{22}$ during years without degradation.

A phase-coherent supercontinuum. We experimentally investigate the phase coherence of the supercontinuum generated in

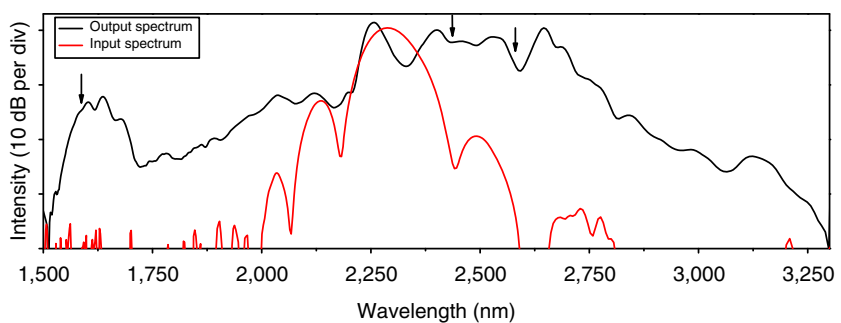

Figure 2 | The spectrum at the input (red) and the output (black) of

the silicon nanowire. The input pulses are centred at 2,290 nm and have a coupled peak power of $225 \mathrm{~W}$. Their spectrum is broadened in the silicon photonic wire such that it experimentally spans more than an octave: the $30-\mathrm{dB}$ bandwidth covers from 1,540 to $3,200 \mathrm{~nm}$. The arrows indicate the wavelength position where the phase coherence measurements are performed. the waveguide by beat note measurements with a set of narrow line-width continuous-wave lasers. Such a characterization technique for assessing comb coherence properties is an alternative to that involving a $f-2 f$ interferometer ${ }^{23}$ and it is well documented in the literature, for example refs $3,4,24$. Here it was chosen because our foreseen applications ${ }^{25}$ to molecular spectroscopy do not require self-referencing of the comb. In this characterization, all laser systems, including the continuous wave ones, are freerunning. First, we beat the free-running seed source with a tunable continuous wave OPO (Argos Aculight, line-width $\sim 60 \mathrm{kHz}$ at $500 \mu \mathrm{s})$ at $2,400 \mathrm{~nm}$ on a fast InGaAsSb photodetector (Fig. 3a). We then beat the supercontinuum output with the same OPO (Fig. 3b,c), respectively, tuned at 2,418 and $2,580 \mathrm{~nm}$. We finally beat (Fig. 3d), on a fast InGaAs detector, the supercontinuum with a narrow line-width erbium-doped fibre laser (Koheras AdjustiK E15, NKT Photonics, line-width $0.1 \mathrm{kHz}$ at $100 \mu \mathrm{s})$ at $1,586 \mathrm{~nm}$, far from the seed wavelength. All radiofrequency spectra are recorded with a $100-\mathrm{kHz}$ resolution bandwidth, and a spectrum with a $105-\mathrm{MHz}$ span shows three isolated lines. The strong beat signal at $100 \mathrm{MHz}$ corresponds to the repetition frequency of the fs OPO, while the other two beat notes correspond to the beat signal generated by the continuous wave lasers and the two spectrally closest lines of the frequency comb. The line-width of the beat notes, measured with a $10-\mathrm{kHz}-$ resolution bandwidth (insets in Fig. 3a-d) is limited by the instabilities of the free-running lasers, but it is found to be $\sim 50 \mathrm{kHz}$, without noticeable broadening relative to the fs OPO seed source. The width of the free-running beat notes is the convolution of the width of the two beating laser lines. However, the width of the lines of the free-running femtosecond modelocked Ti-Sapphire laser used to synchronously pump the seed fs $\mathrm{OPO}$ is similar. Stabilizing the system against a radiofrequency reference, such as a caesium clock, is not expected to bring significant line-width reduction: the locking electronics would need a bandwidth that only compensates for slow fluctuations $(\sim 100 \mathrm{~Hz})$ to avoid 'coherence collapse' by multiplication of the phase noise of the radiofrequency reference ${ }^{26}$. We note that our measured line-widths are in full agreement with that of other free-running or radiofrequency-referenced frequency comb systems ${ }^{26}$. Our investigation thus demonstrates the frequency comb structure of the supercontinuum.

Comparison with simulations. The coherence of the supercontinuum can be simulated and such simulations can be used to confirm the frequency comb structure at the probed wavelengths as well as indicating the coherence over the whole bandwidth. The supercontinuum generation can be simulated by solving the generalized nonlinear Schrödinger equation numerically with a split-step Fourier method ${ }^{5}$ (see Methods). The simulation takes the linear propagation loss, the nonlinear phase shift, the threephoton absorption and both the induced absorption and dispersion by the carriers into account. In the simulation the nonlinear parameter $\gamma$ is assumed to be $38(\mathrm{Wm})^{-1}$, the linear propagation loss is assumed to be $0.1 \mathrm{~dB} \mathrm{~cm}^{-1}$ and the threephoton absorption coefficient is assumed to be $0.025 \mathrm{~cm}^{3} \mathrm{GW}^{-2}$ (ref. 27). Figure 4a) shows the evolution of the spectrum of a $225-\mathrm{W}$ peak power, 70 -fs-long pulse as it is propagating along the silicon photonic wire waveguide. The simulated spectrum after $1-\mathrm{cm}$ propagation is shown in Fig. 4b. As shown in Fig. 4b, the simulation agrees very well with the experimental results. The simulation of the spectral evolution of the pulse along the photonic wire length reveals (Fig. 4a) that, in the first millimeter of propagation, self-phase modulation is the primary mechanism for spectral broadening. The spectrum is further broadened into the telecom wavelength range, where the group 
a

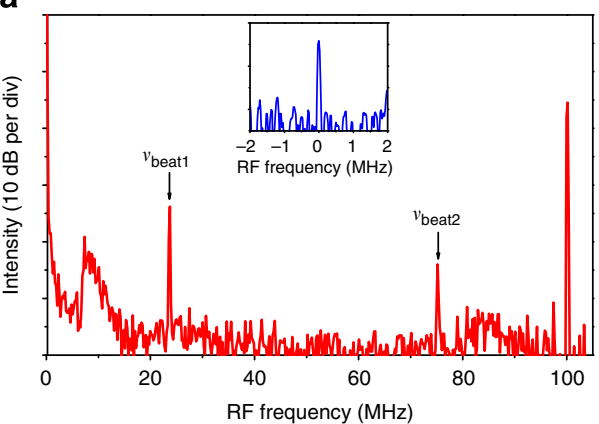

C

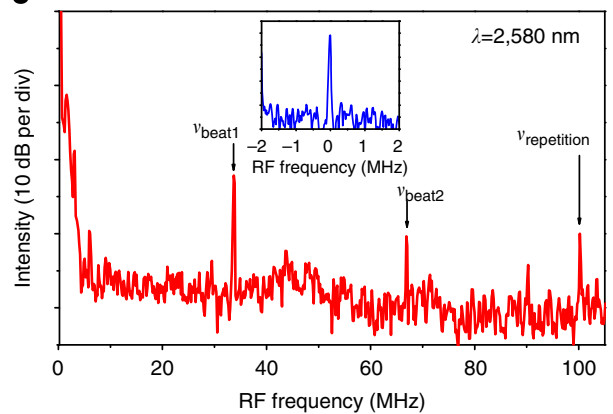

b

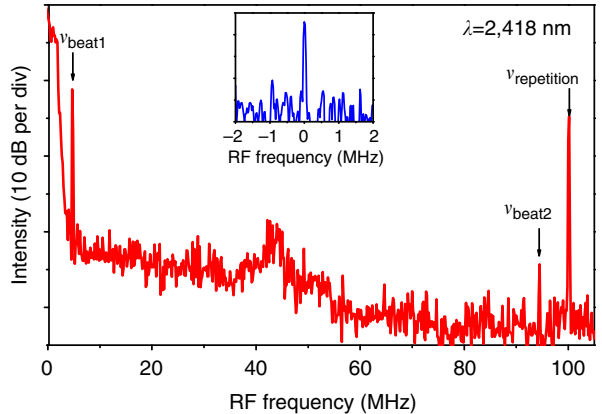

d

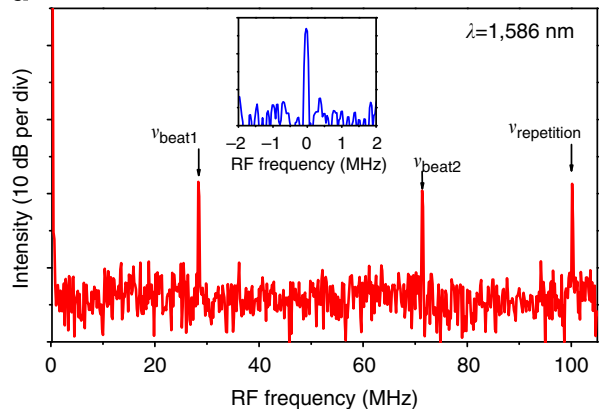

Figure 3 | Experimental radiofrequency spectra of the beat notes. (a) Radiofrequency spectrum of the free-running beat note of the pump pulses and a narrow line-width source at $2,400 \mathrm{~nm}$. (b-d) Free-running beat notes of the spectrally broadened pulses and a narrow line-width source at $\lambda=2,418 \mathrm{~nm}, \lambda=2,580 \mathrm{~nm}$ and $\lambda=1,586 \mathrm{~nm}$, respectively. The insets in the figure show a high-resolution spectrum of the free-running beat notes. The free-running beat notes of the output pulses are measured to be $\sim 50 \mathrm{kHz}$ wide and are not broadened as compared with beat notes measured on the input pulses.
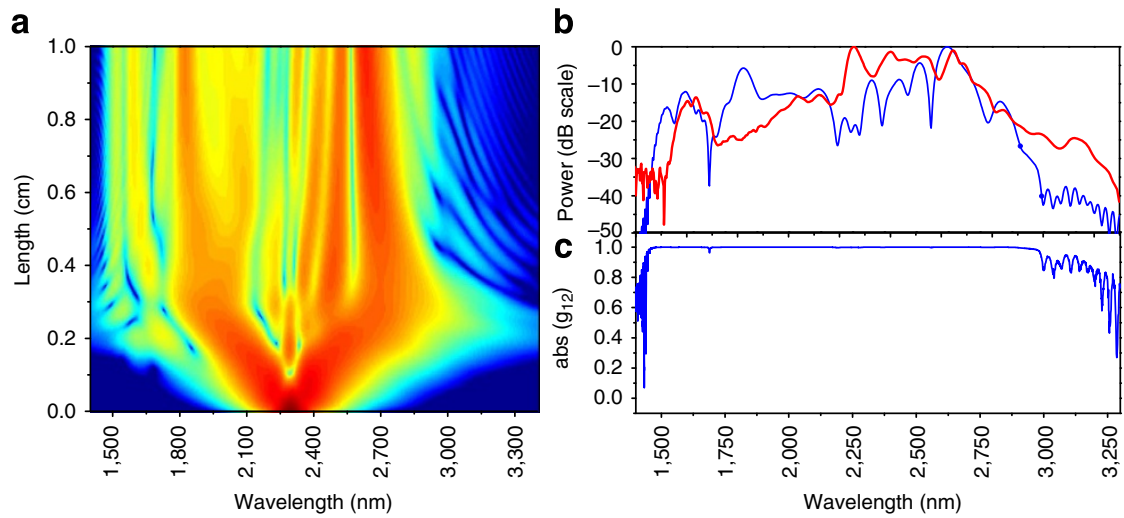

Figure 4 | The simulated spectral broadening and coherence of the pulses. (a) Evolution of the spectral content of the optical pulse along the length of the silicon photonic wire waveguide. (b) Simulated spectra after $1 \mathrm{~cm}$ of propagation in the waveguide (blue) and the measured supercontinuum (red). (c) Simulated coherence as a function of wavelength.

velocity dispersion of the waveguide is normal, through dispersive wave generation ${ }^{28}$. The use of the short pulses favours the processes such as dispersive wave generation and self-phase modulation. Unlike in ref. 29 where longer, ps pulses were used and the spectral broadening primarily results of amplification of background noise (modulation instability), the nonlinear process of dispersive wave generation and self-phase modulation maintain the coherence in the pulse.

The coherence of the supercontinuum can be simulated by including shot noise at the input. The noise $E_{\text {noise }}(t)$ at the input is assumed to be a random variable with a stochastic distribution $\left\langle E_{\text {noise }}(t) E_{\text {noise }}(t+\tau)\right\rangle=\frac{h v}{2} \delta(t)$, with $h$ the Planck constant and $v$ the frequency of the photons, and analysing an ensemble of simulated supercontina ${ }^{30}$. The first-order coherence function

$$
g_{12}(\omega)=\frac{\left|\left\langle E_{i}(\omega) E_{j}(\omega)\right\rangle_{i \neq j}\right|}{\sqrt{\left\langle\left|E_{i}(\omega)\right|^{2}\right\rangle\left\langle\left|E_{j}(\omega)\right|^{2}\right\rangle}}
$$

is calculated for an ensemble of 100 spectra and is shown in Fig. 4c. The coherence is close to unity over the whole spectrum, indicating that the generated supercontinuum is coherent over its entire bandwidth.

To emphasize the comb structure of the supercontinuum spectrum, which results from the pulse-to-pulse coherence, the spectrum of the pulse train at the output of the chip was 


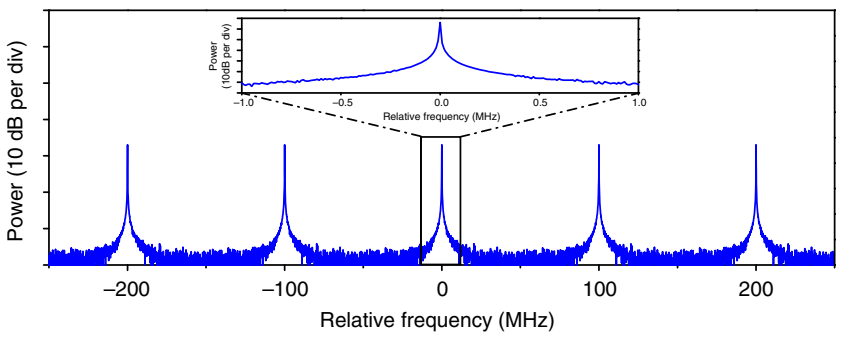

Figure 5 | A simulated high-resolution spectrum of the silicon wire

output. The spectrum of the supercontinuum frequency comb is simulated over a $500-\mathrm{MHz}$ bandwidth in the vicinity of $1,586 \mathrm{~nm}(198 \mathrm{THz})$. It reveals comb lines separated by $100 \mathrm{MHz}$ in the. A high-resolution $(10 \mathrm{kHz})$ inset around a comb line is also shown.

simulated with a resolution of $10 \mathrm{kHz}$ in a narrow band interval. The spectrum is simulated by first generating a set of pulses including the input shot noise, but excluding timing jitter and residual intensity noise, as discussed above. These pulses were stacked together in a pulse train with a repetition frequency of $100 \mathrm{MHz}$. The Fourier transform was calculated to generate the spectrum of the pulse train (see Supplementary Methods and Supplementary Fig. 3 for details). Figure 5 shows the spectrum of a train of 1,000 pulses, calculated in a $500-\mathrm{MHz}$ interval at $1,586 \mathrm{~nm}$. The independent comb lines can clearly be seen. The inset of Fig. 5 shows one individual comb line sampled with a resolution of $10 \mathrm{kHz}$ by calculating the spectrum of a pulse train consisting of 10,000 pulses. Similar simulations were performed in an interval at 2,418 and $2,580 \mathrm{~nm}$, confirming the comb structure of the supercontinuum (see Supplementary Fig. 4). In the simulations, the width of the comb lines is only limited by the time window used.

Interestingly, such nonlinear processes in silicon wires can be harnessed with other mid-infrared ultrashort pulse pump laser systems of different wavelength than the OPO employed here. For example, our simulations (Supplementary Discussion and Supplementary Fig. 5) indicate that a thulium-doped modelocked fibre laser can be used as well to generate a phase-coherent octave-spanning supercontinuum in a dispersion-engineered silicon waveguide.

\section{Discussion}

Using a silicon nanowire on a chip, we have demonstrated an octave-spanning frequency comb spanning from the telecom wavelength window $\sim 1,500 \mathrm{~nm}$ to the mid-infared wavelength range at $3,300 \mathrm{~nm}$. Such frequency comb is readily suitable for direct frequency comb spectroscopy, particularly for dual-comb spectroscopy with, for example, adaptive sampling ${ }^{25}$. Improved dispersion engineering could potentially extend the supercontinuum over the whole transparency window of the SOI platform (1,100-4,000 nm), limited by the buried oxide. Even broader bandwidths could be further obtained up to $5,500 \mathrm{~nm}$ with silicon on sapphire waveguide platforms ${ }^{31,32}$. By using waveguide designs where the buried oxide is removed ${ }^{14,15,33}$, the entire silicon transparency window (up to $8,500 \mathrm{~nm}$ ) could be covered. As many molecules have strong rovibrational lines in the mid-infrared range, such developments would contribute in expanding the intriguing capabilities of molecular spectroscopy with frequency combs to the molecular fingerprint region. Such broadband supercontinua may also lead to self-referenced mid-infrared frequency comb systems, as needed for precision measurements in frequency metrology and in some implementations of direct frequency comb spectroscopy ${ }^{1}$. The rapid progress in the development of miniaturized mid-infrared frequency comb generators, as reported for instance with quantum cascade lasers 2,4 or high-quality factor microresonators ${ }^{34}$, might lead to an entirely new strategy for a compact source of ultrashort pulses in the future. Our work would then represent an essential building block paving the road for an octave-spanning frequency comb entirely generated on a chip. Such prospect would be of interest to, for example, chemical sensing, calibration of astronomical spectrographs, environmental monitoring or free-space communications.

\section{Methods}

Description of the mid-infrared frequency comb seed source. The frequency comb generator that seeds the silicon waveguide is a home-made femtosecond synchronously pumped OPO. Its design and characterization are described in ref. 19. Here we just reproduce the details that are useful for the description of the present experiment. The pump source of the OPO is a Kerr-lens mode-locked Ti:sapphire oscillator with a repetition frequency of $100 \mathrm{MHz}$, an average power of $1 \mathrm{~W}$, a central wavelength of $790 \mathrm{~nm}$ and a pulse duration of $20 \mathrm{fs}$. The nonlinear crystal of the OPO is made of MgO:PPLN with a fan-out grating interaction length of $l=500 \mu \mathrm{m}$. The OPO cavity is a dispersion-controlled four-mirror standingwave design with two planoconcave mirrors and four plane mirrors. We tune the central wavelength of the idler of the OPO to $2,290 \mathrm{~nm}$. The average output power is $35 \mathrm{~mW}$. The idler spectrum is shown in Fig. 2b. We measure the pulse duration with a home-made autocorrelator based on two-photon absorption in a InGaAs photodetector. The autocorrelation (Supplementary Fig. 1) reveals a pulse duration of $72 \mathrm{fs}$, assuming a $\operatorname{sech}^{2}$ profile.

Simulations. The spectral evolution of the pulses along the waveguide is simulated by solving the generalized nonlinear Schrodinger equation numerically using a split-step approach ${ }^{35}$. We solve

$$
\begin{aligned}
\frac{\partial E(z, t)}{\partial z}= & i \sum_{k \geq 2} i^{k} \frac{\beta_{k}}{k !} \frac{\partial^{k} E}{\partial t^{k}}-\frac{\alpha_{l}}{2} \mathrm{E}-\frac{\alpha_{3 e f f}}{2}|E|^{4} E-\alpha_{c}(1+\mu i) E \\
& +i \gamma\left(1+\frac{i}{\omega_{0}} \frac{\partial}{\partial t}\right) E \int_{-\infty}^{t} R\left(t-t^{\prime}\right)\left|E\left(z, t^{\prime}\right)\right|^{2} d t^{\prime}
\end{aligned}
$$

Here $E(z, t)$ is the envelope of the electric field of the short pulses, $\beta_{\mathrm{k}}$ is the kth order dispersion coefficient, $\alpha_{1}$ the linear propagation loss, $\alpha_{3 \text { eff }}$ the effective third-order absorption coefficient, $\alpha_{c}$ the free carrier absorption coefficient, $\mu$ takes the free carrier dispersion in account, $\gamma$ is the nonlinear parameter of the waveguide, while the integral takes in account the fractional Raman response. The effective third-order absorption coefficient can be calculated as $\alpha_{3 e f f}=\frac{n_{g}^{2} \alpha_{3}}{n^{2} A_{5 e f f}^{2}}$ (ref. 36), where $\alpha_{3}$ is the third-order nonlinear absorption coefficient in silicon of $\sim 2.5 \times 10^{-26} \mathrm{~m}^{3} \mathrm{GW}^{-2}$ (refs 27,37 ) and $A_{5 \text { eff }}=0.5 \mu \mathrm{m}^{2}$ the fifth-order mode area. The carrier-induced absorption coefficient is proportional to the carrier density $N_{c}$, such that $\alpha_{c}=\sigma N_{c}$, where $\sigma=2.77 \times 10^{-21} \mathrm{~m}^{2}$ (ref. 38), while $\mu=\frac{2 k_{c}}{\sigma \omega_{0} c}$ with $k_{\mathrm{c}}=1.35 \times 10^{-27} \mathrm{~m}^{3}$ (ref. 38). The evolution of the carrier density itself can be calculated as $\frac{\partial N_{c}(z, t)}{\partial t}=\frac{2 \pi \alpha_{3 e f f}}{3 h \omega} \frac{|E(z, t)|^{6}}{A_{\text {eff }}}-\frac{N_{c}(z, t)}{\tau}$ (ref. 38), where $h$ is Planck's constant and $\tau$ the carrier lifetime, estimated to be 1 ns (ref. 39). It was assumed that the pulse was a hyperbolic secant with a full-width at half-maximum of $70 \mathrm{fs}$.

\section{References}

1. Schliesser, A., Picqué, N. \& Hänsch, T. W. Mid-infrared frequency combs. Nat. Photon. 6, 440-449 (2012).

2. Hugi, A., Villares, G., Blaser, S., Liu, H. \& Faist, J. Mid-infrared frequency comb based on a quantum cascade laser. Nature 492, 229-233 (2012).

3. Wang, C. Y. et al. Mid-infrared optical frequency combs at $2.5 \mu \mathrm{m}$ based on crystalline microresonators. Nat. Commun. 4, 1345 (2013).

4. Burghoff, D. et al. Terahertz laser frequency combs. Nat. Photon. 8, 462-467 (2014).

5. Dudley, J. M., Genty, G. \& Coen, S. Supercontinuum generation in photonic crystal fiber. Rev. Mod. Phys. 78, 1135-1184 (2006).

6. Marandi, A. et al. Mid-infrared supercontinuum generation in tapered chalcogenide fiber for producing octave-spanning frequency comb around 3 $\mu \mathrm{m}$. Opt. Express 20, 24218-24225 (2012).

7. Rudy, C. W., Marandi, A., Vodopyanov, K. L. \& Byer, R. L. Octave-spanning supercontinuum generation in in situ tapered $\mathrm{As}_{2} \mathrm{~S}_{3}$ fiber pumped by a thulium-doped fiber laser. Opt. Lett. 38, 2865-2868 (2013).

8. Lee, K. et al. Mid-infrared frequency combs from coherent supercontinuum in chalcogenide nanospike. Opt. Lett. 39, 2056-2059 (2014).

9. Granzow, N. et al. Mid-infrared supercontinuum generation in As2S3-silica 'nano-spike' step-index waveguide. Opt. Express 21, 10969-10977 (2013). 
10. Shabahang, S. et al. CLEO: 2013, OSA Technical Digest. Paper SW3I.2 (Optical Society of America, San Jose, CA, USA, 2013) http://dx.doi.org/ 10.1364/CLEO_SI.2014.SW3I.2.

11. Phillips, C. R. et al. Supercontinuum generation in quasi-phase-matched $\mathrm{LiNbO}_{3}$ waveguide pumped by a Tm-doped fiber laser system. Opt. Lett. 36, 3912-3914 (2011).

12. Phillips, C. R., Pelc, J. S. \& Fejer, M. M. Parametric processes in quasiphasematching gratings with random duty cycle errors. J. Opt. Soc. Am. B 30, 982-993 (2013).

13. Halir, R. et al. Ultrabroadband supercontinuum generation in a CMOScompatible platform. Opt. Lett. 37, 1685-1687 (2012).

14. Soref, R. Mid-infrared photonics in silicon and germanium. Nat. Photon. 4, 495-497 (2010).

15. Jalali, B. Nonlinear optics in the mid-infrared. Nat. Photon. 4, 506-508 (2010).

16. Lau, R. et al. Octave-spanning mid-infrared supercontinuum generation in silicon nanowaveguides. Opt. Lett. 39, 4518-4525 (2014).

17. Bogaerts, W. et al. Nanophotonic waveguides in silicon-on-insulator fabricated with CMOS technology. J. Lightw. Technol. 23, 401-412 (2005).

18. Bristow, A. D., Rotenberg, N. \& Van Driel, H. M. Two-photon absorption and Kerr coefficients of silicon for 850-2200 nm. Appl. Phys. Lett. 90, 191104 (2007).

19. Kumar, S. C. et al. Few-cycle, broadband, mid-infrared optical parametric oscillator pumped by a 20-fs Ti:sapphire laser. Laser Photon. Rev. 8, L86-L91 (2014).

20. $\mathrm{Pu}$, M., Liu, L., Ou, H., Yvind, K. \& Hvam, J. M. Ultra-low-loss inverted taper coupler for silicon-on-insulator ridge waveguide. Opt. Commun. 283, 3678-3682 (2010).

21. Colinge, J. Silicon-on-Insulator Technology: Materials to VLSI (Kluwer Academic Publishers, 2004).

22. Hochberg, M. \& Jones, T. B. Towards Fabless silicon photonics. Nat. Photon. 4, 492-494 (2010).

23. Udem, T., Holzwarth, R. \& Hänsch, T. W. Optical frequency metrology. Nature 416, 233-237 (2002).

24. Gohle, C. et al. A frequency comb in the extreme ultraviolet. Nature 436, 234-237 (2005).

25. Ideguchi, T. et al. Adaptive real-time dual-comb spectroscopy. Nat. Commun. 5, 3375 (2014).

26. Diddams, S. A., Ye, J. \& Hollberg, L. in Femtosecond Optical Frequency Comb: Principle, Operation and Applications (eds Ye, J. \& Cundiff, S. T.) 225-262 (Springer, 2005).

27. Pearl, S., Rotenberg, N. \& Driel, H. M. Three photon absorption in silicon for 2300-3300 nm. Appl. Phys. Lett. 93, 131102 (2008).

28. Cristiani, I., Tediosi, R., Tartara, L. \& Degiorgio, V. Dispersive wave generation by solitons in microstructured optical fibers. Opt. Express 12, 124-135 (2004).

29. Kuyken, B. et al. Mid-infrared to telecom-band supercontinuum generation in highly nonlinear silicon-on-insulator wire waveguides. Opt. Express 19, 20172-20181 (2011)

30. Ruehl, A. et al. Ultrabroadband coherent supercontinuum frequency comb. Phys. Rev. A 84, 11806-11811 (2011).

31. Baehr-Jones, T. et al. Silicon-on-sapphire integrated waveguides for the mid-infrared. Opt. Express 18, 12127-12135 (2010).

32. Li, F. et al. Low propagation loss silicon-on-sapphire waveguides for the mid-infrared. Opt. Express 19, 15212-15220 (2011).
33. Soref, R., Emelett, A. \& Buchwald, W. R. Silicon waveguided components for the long-wave infrared region. J. Opt A Pure Appl. Opt. 8, 840-853 (2006).

34. Griffith, A. G. et al.Silicon-chip mid-infrared frequency comb generation. Preprint at http://arXiv.org/abs/1408.1039 (2014).

35. Yin, L., Lin, Q. \& Agrawal, G. P. Soliton fission and supercontinuum generation in silicon waveguides. Opt. Lett. 32, 391-393 (2007).

36. Husko, C. et al. Non-trivial scaling of self-phase modulation and three-photon absorption in III-V photonic crystal waveguides. Opt. Express 17, 22442-22451 (2009).

37. Gai, X. et al. Nonlinear absorption and refraction in crystalline silicon in the mid-infrared. Laser Photon. Rev. 7, 1054-1064 (2013).

38. Lin, Q., Painter, O. J. \& Agrawal, G. P. Nonlinear optical phenomena in silicon waveguides: modeling and applications. Opt. Express 15, 16604-16644 (2007)

39. Boyraz, O., Koonath, P., Raghunathan, V. \& Jalali, B. All optical switching and continuum generation in silicon waveguides. Opt. Express 12, 4094-4102 (2004).

\section{Acknowledgements}

B.K. acknowledges the special research fund of Ghent University (BOF), for a post doctoral fellowship. We are grateful to Dr Antonin Poisson and Dr Clément Lafargue for experimental support. This work was partly carried out in the framework of the Methusalem project 'Smart Photonic Chips' and the FP7-ERC-INSPECTRA, FP7-ERC MIRACLE and FP7-ERC-Multicomb (Advanced Investigator Grant 267854) projects.

\section{Author contributions}

B.K. performed the numerical dispersion design calculations with guidance from R.B. and G.R., J.V.C. and P.V. supervised the waveguide device fabrication process. B.K., T.I., S.H. and M.Y. performed the supercontinuum generation experiment as well as the beat note experiment with guidance and supervision from T.W.H. and N.P. T.I., S.H. and M.Y. performed the autocorrelation experiment under the supervision of N.P. B.K., F.L and S.C. performed the simulations on the coherence. B.K. drafted the manuscript. All authors provided comments and suggestions for improvements.

\section{Additional information}

Supplementary Information accompanies this paper at http://www.nature.com/ naturecommunications

Competing financial interests: The authors declare no competing financial interests.

Reprints and permission information is available online at http://npg.nature.com/ reprintsandpermissions/

How to cite this article: Kuyken, B. et al. An octave-spanning mid-infrared frequency comb generated in a silicon nanophotonic wire waveguide. Nat. Commun. 6:6310 doi: $10.1038 /$ ncomms7310 (2015)

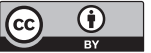

This work is licensed under a Creative Commons Attribution 4.0 International License. The images or other third party material in this article are included in the article's Creative Commons license, unless indicated otherwise in the credit line; if the material is not included under the Creative Commons license, users will need to obtain permission from the license holder to reproduce the material. To view a copy of this license, visit http://creativecommons.org/licenses/by/4.0/ 